\title{
Intelligent Detection Method of Yarn Hairiness Using CenterNet-CBAM
}

\author{
JialuTang $^{\mathrm{a}, 1}$, Zhongliang Yang ${ }^{\mathrm{a}}$, Xinyu Zhang ${ }^{\mathrm{a}}$, Xingli Jia ${ }^{\mathrm{a}}$, Song Zhang ${ }^{\mathrm{b}}$, \\ Qingqi Dong ${ }^{\mathrm{c}}$ \\ ${ }^{a}$ College of Mechanical Engineering, Donghua University, Shanghai 201620, China \\ ${ }^{b}$ Department of Materials, University of Manchester, Manchester M13 9PL, United \\ Kingdom

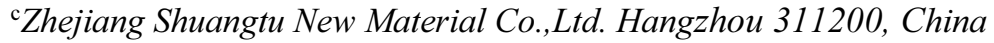

\begin{abstract}
The detection of yarn hairiness is essential in the production of chemical fiber yarn packages, and it is difficult to detect them because of their small features, which are easy to be missed and confused with another non-defective feature broken ends. To detect defects accurately and efficiently in the appearance of yarn packages, a CenterNet defect detection algorithm (CenterNet-CBAM) combining with attention mechanism is proposed. Two types of confusing target images, "yarn hairiness" and "broken ends", are collected, and an object detection model based on CenterNet-CBAM is constructed, and the Recall of CenterNet-CBAM in the two categories of "yarn hairiness" and "broken ends" is $90.20 \%$ and $85.42 \%$, Precision is $93.88 \%$ and $93.48 \%$, $\mathrm{AP}$ is $90.91 \%$ and $90.93 \%$, and MAP is $90.92 \%$ for the two categories, respectively, which were better than CenterNet and YOLOv4, which verified the effectiveness of the experimental method.
\end{abstract}

Keywords. yarn packages detection, object detection, attention mechanism, yarn hairiness

\section{Introduction}

At present, the intelligent detection of yarn hairiness faces a problem, "yarn hairiness" and "broken ends" are not easy to be distinguished, which easily leads to wrong detection. To address the above problems, this paper proposes a yarn hairiness object detection algorithm CenterNet-CBAM combining attention mechanism. Compared with CenterNet and YoloV4 object detection algorithms, the method in this paper achieves a higher detection effect of yarn hairiness and can effectively distinguish yarn hairiness from broken ends.

The task of Object Detection is to find all the objects to be detected in an image, determine their classes and location. Two-stage algorithm Faster R-CNN [1]proposed in 2015 is a typical algorithm in the defect detection task, and achieves good results in fabric defect detection by adjusting the region recommendation network (RPN). In 2016, the YOLO $^{[2]}$ achieved classification and localization in one step, combining Depthwise Separable Convolution with YOLO model, fabric defect recognition accuracy reached

\footnotetext{
${ }^{1}$ Corresponding Author.
} 
$95.8 \%{ }^{[3]}$. The above studies are aimed at fabric defect detection, while no literature has been reported in the field of yarn packages surface detection.

The surface defects of yarn packages mainly include yarn hairiness, holes and stains, while broken end are ends of chemical fiber, which are not surface defects. Wang et al. ${ }^{[4]}$ used the maximum pooling layer instead of the fully connected layer to make a lightweight improvement to the AlexNet network, which mainly focused on the obvious defect features and did not detect the yarn hairiness that are difficult to be directly observed by human eyes. To address this problem, Zhang et al. added deconvolution and feature fusion to the $\mathrm{SSD}^{[5]}$ model to classify and detect various types of yarn hairiness in yarn packages, but did not distinguish between yarn hairiness and broken ends. In 2019, Zhou et al. ${ }^{[6]}$ proposed the CenterNet algorithm, which uses an anchor-free detection method to transform the object detection problem into a local offset computation problem, and because of this property, it can be used in the detection of small objects such as Maize Tasse ${ }^{[7]}$, diabetic retinopathy ${ }^{[8]}$, and other small objects detection, which can be used in yarn surface defect detection.

Classical convolution mainly focuses on spatial local feature extraction without filtering global and channel image information. To avoid information overload while capturing more important feature information in the image, and to improve the efficiency and accuracy of task processing, the Attention Mechanism is proposed. The attention mechanism is similar to the human attention behavior, highlighting the key information and weakening the secondary information.

The Convolutional Block Attention Module (CBAM) $)^{[9]}$ which combines spatial and channel attention mechanism, can improve the learning effect of the network, and the ablation experiment shows that the Top-1 Error of ResNet50 network with CBAM decreases about $2 \%$ in the identification of hot rolled steel surface defect detection ${ }^{[10]}$, fabric defect detection. Since the embedded CBAM attention mechanism can effectively improve the effect of image classification, if CBAM is combined with the object detection algorithm, it will further improve the accuracy of yarn hairiness detection.

\section{Methods}

\subsection{Visual acquisition platform}

In the surface defect detection system, hardware equipment such as cameras and light sources are the basic support. The yarn is placed on an experimental inspection platform that can be rotated, and the industrial microscope camera is fixed and connected to the filament rod by clamps. The filament rod is clamped on a cross slide, and when the inspection position needs to be changed, the cross slide is used to adjust in both vertical and front-back directions to finally achieve precise displacement. The image capture process uses a motor to drive the bearing to rotate and change the angle to update the image. 


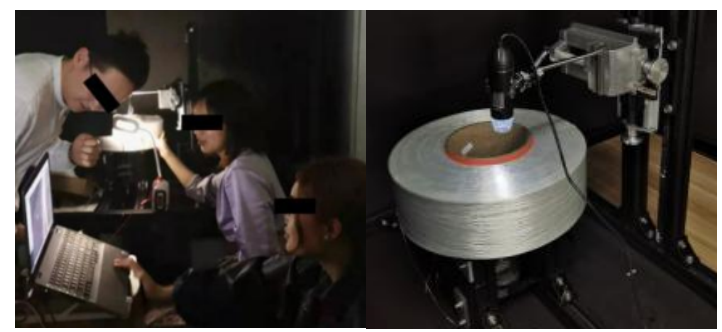

Figure 1. Image acquisition experimental platform

The light source is provided at a specific angle to illuminate the yarn hairiness, weakening the effect of reflections on the surface of the yarn on the defective features, and obtaining a picture with bright features and dark background as shown in the figure 2. The following experiments will determine the best camera mounting position and machine vision light source configuration.

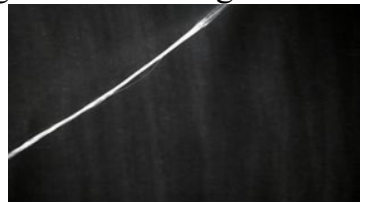

(a)Broken end

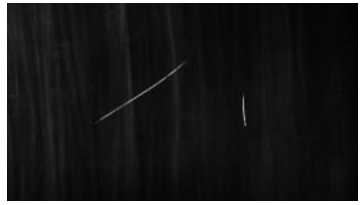

(b) Yarn hairiness

Figure 2. Broken end and yarn hairiness taken by industrial microscope

\subsection{Equipment options}

Choose the focal length $60 \mathrm{~mm}$ magnifying lens, optical magnification $1.2 \times \sim 1.8 \times$, pixel $1920 \mathrm{px} \times 1080 \mathrm{px}$. Camera use CMOS 1/2.7" OV2701 sensor, target surface size $6.6 \times 5.2 \times 9.4(\mathrm{~mm})$. The screen size control is the maximum frame that can be shot to defects. According to the lens parameters, adjust the shooting distance, change the target object shooting field of view.

The object detection process requires the detection of yarn hairiness with a diameter of $3 \mu \mathrm{m}$, and the actual size is $30 \mu \mathrm{m}$ after the feature is enlarged by light irradiation. The minimum number of detected pixels was set to 2 pixels. The maximum detection size in horizontal and vertical directions is $28.8 \mathrm{~mm}$ and $16.2 \mathrm{~mm}$ respectively. The object distance from the lens of the examined defect is $261.8 \mathrm{~mm}$

\subsection{Defect detection algorithm model}

In this paper, CenterNet model is used, and its algorithm flow is shown in Figure 3. The characteristics of broken end and yarn hairiness are thick in the middle and thin on both sides, which correspond to the heat map recognition mechanism of CenterNet algorithm, with obvious local offset features and weak edge features. The image is passed into the Resnet50 convolutional network to get the heat map, and the peak point of the heat map is the local offset, and the local offset position and the size of the predicted frame are accurately located by local offset prediction and width-height prediction. 


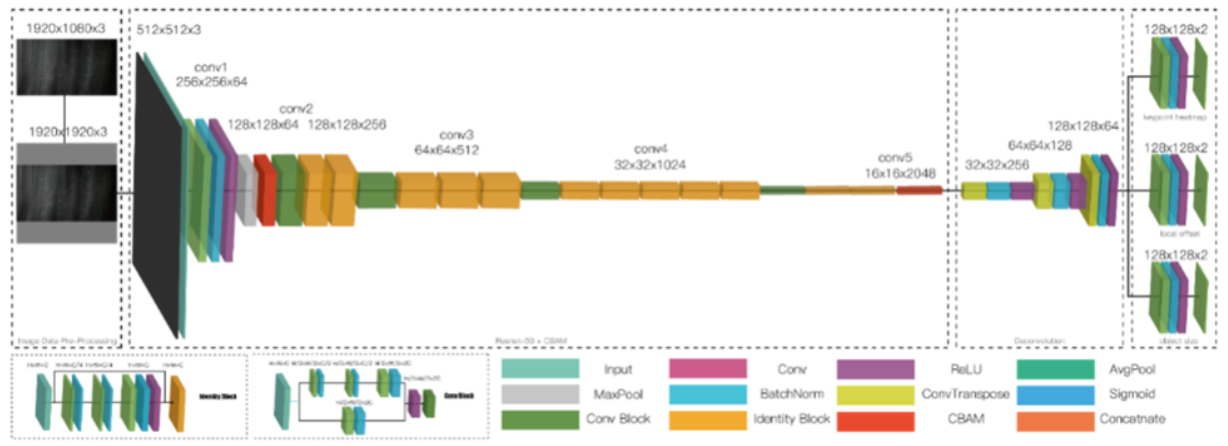

Figure 3. CenterNet-CBAM model framework

Step1: Image pre-processing. For using the pre-trained model, the original image size of $1920 \times 1080 \times 3$ was reduced to $512 \times 512 \times 3$ by padding and resizing process while the aspect ratio was kept constant.

Step2: Convolutional feature extraction. The Resnet50 feature extraction network is used to transform the information in the images into feature arrays by multiple convolution operations. To avoid the accuracy degradation caused by the deepening of the network, The CBAM module is introduced after the maxpool layer of conv1 and the Resnet50 feature extraction network conv5.

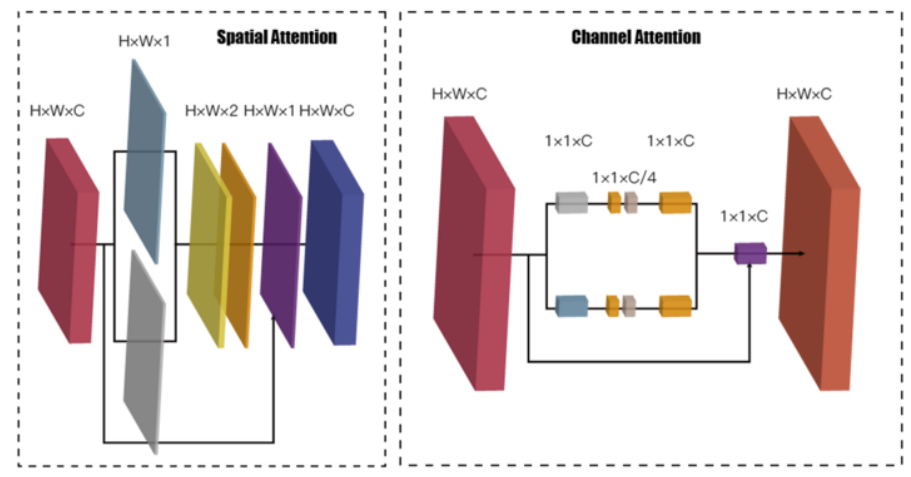

Figure 4. Spatial Attention and Channel Attention in CBAM

Step3: Feature decoding. The image information obtained in the feature extraction network is used to generate the key-point heatmap, local offset, object size prediction results, as shown in Figure 5. The heat map predicts the feature points in the image and the corresponding defect types. For local offset prediction, local offset coordinates (x,y) of the object are output; for object size prediction, the width-height of each type of target defect is exported.

Step4: Generate detection results. All prediction results are processed with Nonmaximum suppression (NMS) to avoid repeated box selection on the same object, and the processed output results are marked on original image for display. 


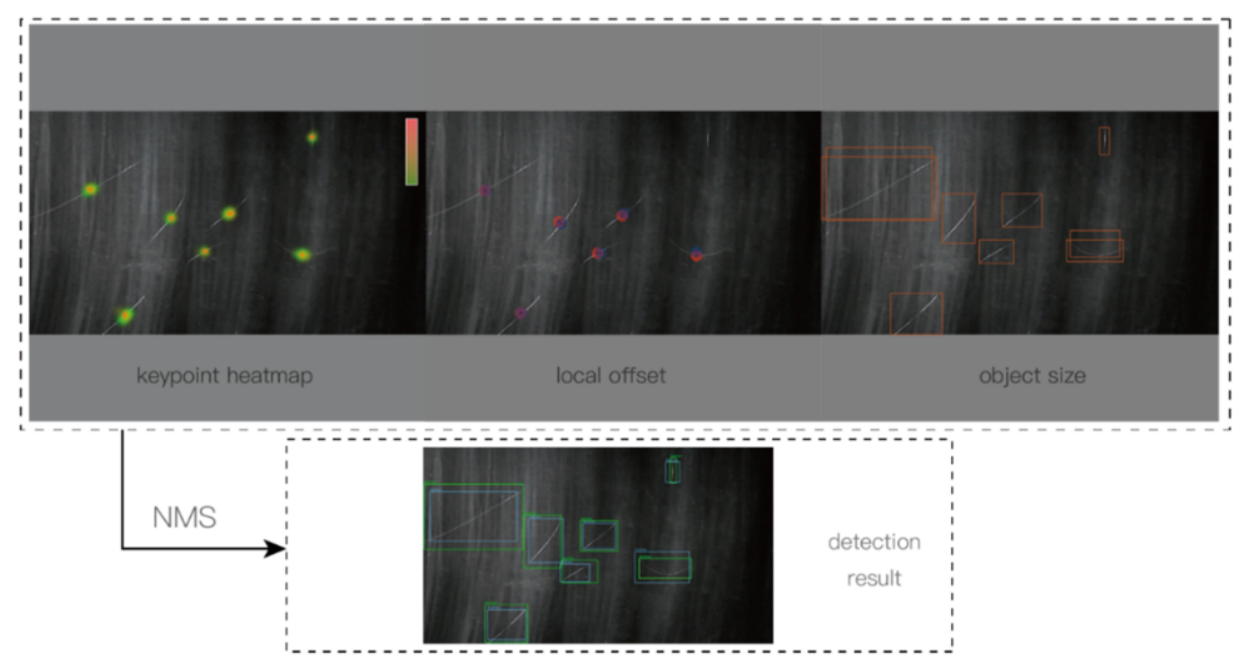

Figure 5. Feature decoding and detection results generation

\subsection{Evaluation indicators}

The model was evaluated using the yarn defect test dataset to calculate the model Precision, Recall, AP, MAP values for broken end and yarn hairiness, and MAP values for both categories.

- $\quad \mathrm{TP}, \mathrm{TN}, \mathrm{FP}, \mathrm{FN}$

The four values represent the relationship between positive and negative sample true values and predicted values. True and False refers to the actual outcome, and Positive and Negative indicate whether the defect is detected or not.

- $\operatorname{Precision}(\mathrm{P})$

In this model, the accuracy rate of defects is the proportion of images predicted to be defective and correct to all images predicted to be defective, calculated as follows.

$$
\mathrm{P}=\frac{T P}{T P+F P}
$$

- $\quad$ Recall (R)

Recall is the percentage of images that are predicted to be defective and correct to all defective images, calculated as follows.

$$
\mathrm{R}=\frac{T P}{T P+F N}
$$

- AP

Calculating AP values first requires plotting Precision-Recall (P-R) curves, counting the prediction frames retained in this picture after non-maximal suppression (NMS) and their corresponding label types and confidence levels.

$$
\mathrm{AP}=\int_{0}^{1} p(\mathrm{r}) d_{r}
$$

- MAP

For the two categories of broken ends and yarn hairiness separately calculated AP, $\mathrm{k}$ is the detection object category.

$$
M A P=\frac{\sum_{i=1}^{k} A P_{i}}{k}
$$




\section{Experiment}

The polyester yarn packages to be tested has an outer diameter of $425 \mathrm{~mm}$, an inner diameter of $140 \mathrm{~mm}$, and a thickness of $123 \mathrm{~mm}$. The polyester fiber wound on a paper tube to make a yarn package, which has good stability and no complete stretching. The surface is smooth and shiny, and the main color is white. As the yarn hairiness defects belong to highly reflective objects, sensitive to lights. Through the light source, the measured product defects part will produce high brightness, and the background environment difference is obvious. To avoid stray light and reflection in the shooting environment, an image acquisition experimental platform was built to create an all-black environment.

\subsection{Data acquisition}

According to the smooth surface of the product, high reflective characteristics of the light source scheme and conduct experiments, and then obtain the best light source scheme.

The process of labeling the yarn hairiness image is shown in the figure 6 , the defects are classified into two categories "broken ends" and "yarn hairiness", and the classification criteria are determined according to the diameter of the inspected defects. After labeling, select the export form of Pascal VOC.

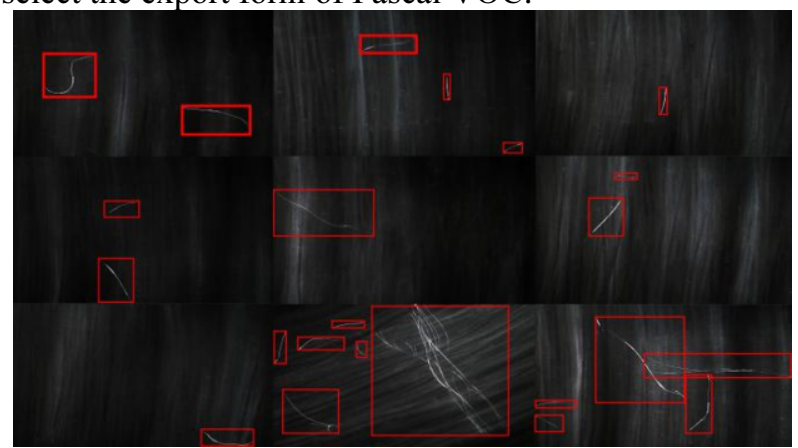

Figure 6. Yarn hairiness label

According to the type and presence of defects on the yarn, the images collected by the industrial microscope were screened to remove samples with poor images, such as out-of-focus and over-exposure, and the final valid images were classified as $\mathrm{YH}, \mathrm{BE}$, and Perfect, respectively, with broken end, yarn hairiness, and no defects. About 500 images were collected for each class, totaling 1614 images.

Table 1. Type and number of pictures taken

\begin{tabular}{ccccc}
\hline Dataset & Perfect & YH & BE & Total \\
\hline Samples & 501 & 543 & 570 & 1614 \\
\hline
\end{tabular}

\subsection{Algorithm Comparison}

The experiments compare 3 algorithms, CenterNet-CBAM, CenterNet, YoloV4 to select the optimal algorithm. All use pretrained models with the training parameters shown in Table 2.

Table 2. training parameters for models

\begin{tabular}{cccc}
\hline Training Epoch & Epoch & Batch size & Leaning rate \\
\hline Freezing Epoch & 50 & 8 & $1 \times 10-3$ \\
Unfreezing Epoch & 150 & 8 & $1 \times 10-4$ \\
\hline
\end{tabular}


The network models were trained on Ubuntu (9.3.0), using GPU acceleration. The system hardware are Intel(R) Core (TM) i5-10600KF CPU @ 4.10GHz and 32 GB RAM, NVIDIA GeForce RTX3090 24 GB. The programming language is Python 3.6, mainly implemented using PyTorch platform. Experiments were conducted using 105 randomly selected images from the Yarn dataset as the test set used for this evaluation.

\section{$4 \quad$ Results and discussion}

\subsection{Identification results of CenterNet-CBAM}

The highest MAP value of CenterNet-CBAM model is $90.92 \%$, and the recall of broken end and yarn hairiness are $90.20 \%$ and $85.43 \%$, and the precision of broken end and yarn hairiness are $93.88 \%$ and $93.48 \%$ respectively. From the figure 7 , we can see that the precision of both categories is similar, but the recall of broken end is higher, which proves that the broken ends feature is more obvious and easier to be extracted.
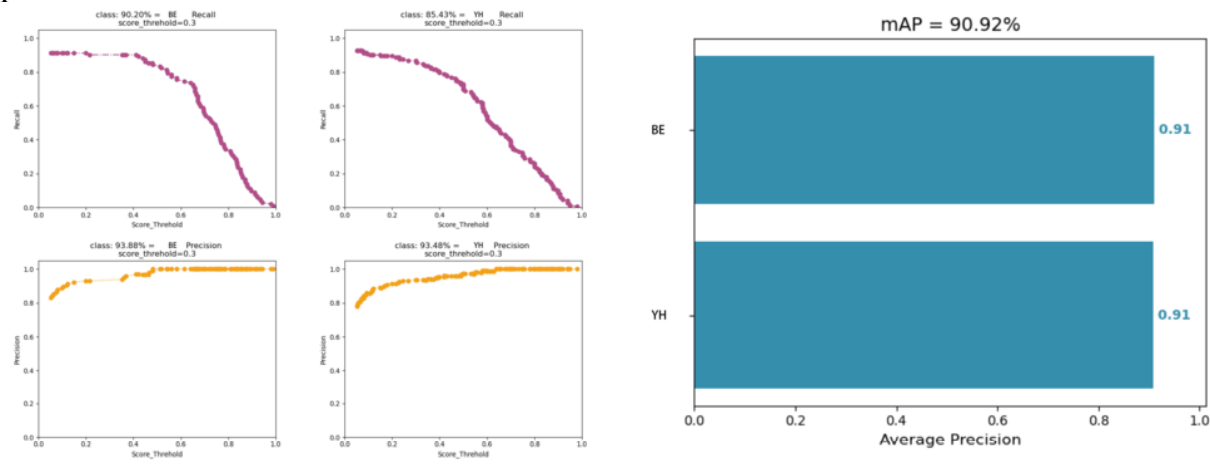

Figure 7. Identification results of CenterNet-CBAM

\subsection{Identification results of CenterNet}

The MAP for defect recognition is $88.86 \%$. the recall of broken end and yarn hairiness are $89.32 \%$ and $68.72 \%$, and the precision of broken end and yarn hairiness are $92.38 \%$ and $93.71 \%$ respectively.
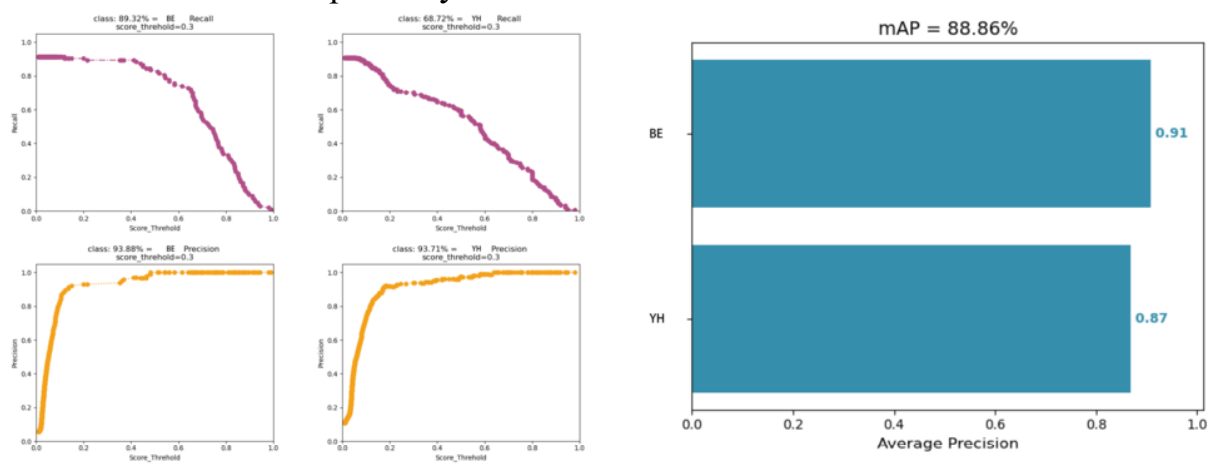

Figure 8. Identification results of CenterNet 


\subsection{Identification results of YOLOv4}

The MAP value of YOLOv4 model is $85.34 \%$, and the Recall of recognition of broken end and yarn hairiness are $74.47 \%$ and $63.86 \%$, respectively, and the precision are $87.50 \%$ and $90.91 \%$, respectively.
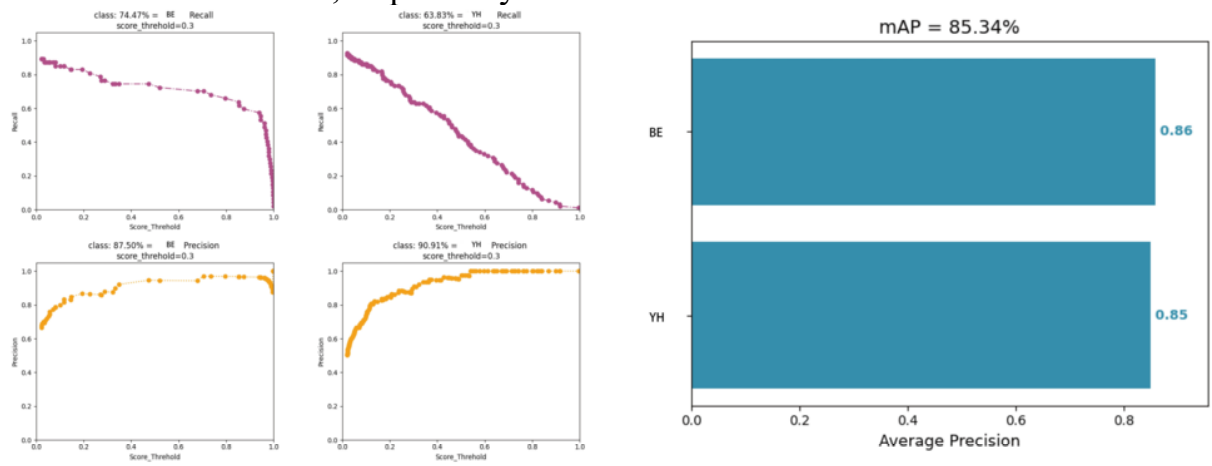

Figure 9. Identification results of YOLOv4

\section{Conclusion}

The recognition results of CenterNet, CenterNet-CBAM, and YOLOv4 algorithms are shown in the figure 10. The blue boxes are Ground truth boxes, the green boxes are Prediction boxes. In the recognition result of CenterNet, the yarn hairiness in the upper right corner is not detected and a miss detection occurs. The IOU of the box selected range is small and the position is not accurate enough. Recognition results of CenterNetCBAM show that all defects are detected, and their locations were more accurate. In YOLOv4, all defects were detected, but due to the high sensitivity of the model, the yarn hairiness are mistakenly identified as broken ends.

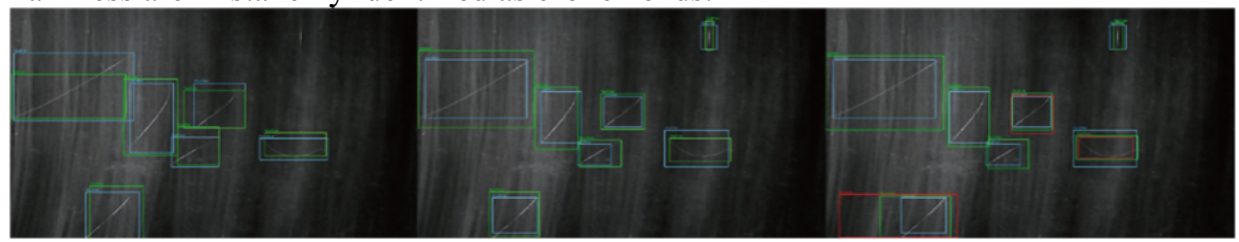

(a)CenterNet

(b)CenterNet-CBAM

(b)YOLOv4

Figure 10. Algorithm performance comparison

The Recall of broken end and broken feather in CenterNet model with added attention mechanism were improved by $0.88 \%$ and $16.7 \%$. The attention mechanism module can extract task-relevant features, suppress task-irrelevant features, redefine the importance of each channel and spatial feature, and achieve a considerable improvement in network performance, model recognition rate, and model convergence speed with a small overhead.

Compared to the YOLOv4 model, the CenterNet model effectively avoids repeat boxing. YOLOv4 object detection algorithm has numerous anchors set on the image first, and the prediction results of the network will be adjusted to obtain the prediction results, and anchors largely improve the detection capability of the network, but it is easy to cause overfitting due to small sample size. 
Table 3. Comparison of algorithm detection results

\begin{tabular}{cccccccc}
\hline Model & $\mathbf{R}_{\mathrm{BE}}$ & $\mathbf{R}_{\mathbf{Y H}}$ & $\mathbf{P}_{\mathrm{BE}}$ & $\mathbf{P}_{\mathrm{YH}}$ & $\mathbf{A P} \mathbf{B E}$ & $\mathbf{A P}_{\mathrm{YH}}$ & $\mathbf{M A P}$ \\
\hline CenterNet-CBAM & 90.20 & 85.42 & 93.88 & 93.48 & 90.91 & 90.93 & 90.92 \\
CenterNet & 89.32 & 68.72 & 92.38 & 93.71 & 90.92 & 86.81 & 88.86 \\
YoloV4 & 74.47 & 63.83 & 87.50 & 90.91 & 85.73 & 84.95 & 85.34 \\
\hline
\end{tabular}

\section{References}

[1] Ren S, He K, Girshick R, Sun J J a I N I P S. Faster r-cnn: Towards real-time object detection with region proposal networks[J]. 2015, 28: 91-99.

[2] Redmon J, Divvala S, Girshick R, Farhadi A. You only look once: Unified, real-time object detection; proceedings of the Proceedings of the IEEE conference on computer vision and pattern recognition, F, 2016.

[3] WANG Zexia, CHEN Ge, CHEN Zhenzhong, Surface defect recognition of chemical fiber yarn packages based on improved convolutional neural network. Journal of Textile Research, 2020, 41(04): 39-44.

[4] Krizhevsky A, Sutskever I, Hinton G E J a I N I P S. Imagenet classification with deep convolutional neural networks. 2012, 25: 1097-1105.

[5] Liu W, Anguelov D, Erhan D, Szegedy C, Reed S, Fu C-Y, Berg A C. Ssd: Single shot multibox detector; proceedings of the European conference on computer vision, F, 2016. Springer.

[6] Zhou X, Wang D, Krähenbühl P J a P A. Objects as points. 2019.

[7] Tahira N, Marriam N, Junaid R, Rabbia M, Momina M, Awais M, Farooq A, Jungeun K, Hyukyoon K, Amir H. Detection of Diabetic Eye Disease from Retinal Images Using a Deep Learning Based CenterNet Model. Sensors (Basel, Switzerland), 2021, 21(16).

[8] ZHANG Chenjia, ZHU Lei, YU Lu Review of Attention Mechanism in Convolutional Neural Networks. Computer Engineering and Applications, 2021, 57(20): 64-72.

[9] Hu J, Shen L, Sun G. Squeeze-and-excitation networks; proceedings of the Proceedings of the IEEE conference on computer vision and pattern recognition, F, 2018.

[10] Jiang Peiying, Tao Qingchuan, Ai Mengqin, Steel Surface Defect Image Classification based on Attention Mechanism and Deep Learning. Computer Application and Software, 2021, 38(09): 214-219. 